\title{
An Algorithm for Traffic Grooming in WDM Optical Mesh Networks with Multiple Objectives
}

\author{
Passakon Prathombutr, Jerry Stach and E.K. Park \\ School of Interdisciplinary Computing and Engineering, University of Missouri, Kansas City, MO 64110 \\ E-mail: \{ppadc,stachj,ekpark\}@umkc.edu
}

\begin{abstract}
This paper studies a traffic grooming in wavelength-division multiplexing (WDM) mesh networks for the SONET/SDH streams requested between node pairs. The traffic could be groomed at the access node before converting to an optical signal carried in the All-Optical network. We design a virtual topology with a given physical topology to satisfy multiple objectives and constraints. The grooming problem of a static demand is considered as an optimization problem. The algorithms found in the literatures focus on a single objective either to maximize the performance or to minimize the cost. We propose a Multi-Objective Evolutionary Algorithm to solve a grooming problem that optimizes multiple objectives all together at the same time. In this paper we consider the optimization of three objectives: maximize the traffic throughput, minimize the number of transceivers, and minimize the average propagation delay or average hop counts. The simulation results show that our approach is superior to an existing heuristic approaches for the 6-node benchmark network in an acceptable running time.
\end{abstract}

KEYWORDS - Multi-Objective Evolutionary Algorithm, Grooming, WDM, mesh optical networks, Pareto Optimal.

\section{INTRODUCTION}

A grooming is a process of multiplexing low-speed traffic onto the high-capacity pipe. It is a challenging technology that could cut costs, improve the protection and decrease provisioning time in the Optical network. While the Optical switch can handle up to OC-768, the electronic switching technologies at the access node can only feed the rates much lower than the full capacity of the Optical network. Therefore, the grooming is the right solution to lower this gap.

Literatures on grooming are mainly based on a ring topology. However, the studies in [1] to [4] purposed the grooming in the WDM mesh network. In [1], they formulated a grooming problem as the ILP with the objective to maximize the static traffic throughput. In [2], they studied the grooming that reduces the number of transceivers in optical networks. The studies in [3] and [4] consider a dynamic traffic pattern in a WDM mesh network.

Our research work is on the traffic grooming in WDM mesh networks with static traffic demand that is similar to [1]. It is considered as an optimization problem. The main purpose is to design the virtual topology that optimizes both a performance and a cost. The objectives include a maximization of a throughput as found in [1], a minimization of the network cost like number of transceivers as found in [2] and a minimization of the average propagation delay of the lightpaths. To achieve these objectives, we propose a multi-objective evolutionary algorithm to search for the solution.

The structure of the remainder of this paper is as follows. Section II presents mathematical formulation of the virtual topology design in the traffic grooming problem. Section III explains the Multi-Objective Evolutionary Algorithm in general. Then in Section IV, we propose how to apply the MOEA for the traffic grooming problem. Section V shows the performance of our approach comparing to the heuristics on a 6-node network. The conclusion of our approach and suggestions for the future work are in Section VI.

- This paper is reprinted from a paper published in ICCCN 2003. Proceedings of the $12^{\text {th }}$ International Conference on Computer Communications and Networks, 20-22 Oct. 2003 Pages 405 - 411

- Passakorn has started working at NECTEC since March 1, 1993. He continued his education in Ph.D. program since January 1997 and graduated in February 2004. 


\section{PROBLEM FORMULATION}

With a given physical topology and traffic demand, we classify the traffic grooming into three subproblems: a lightpath routing, a traffic routing and a wavelength assignment subproblems. The lightpath routing subproblem is the routing of lightpaths on the physical topology. The traffic routing subproblem is the routing of low-speed traffic on the virtual topology under the limitation of the number of transceivers. The wavelength assignment subproblem is the assignment of wavelengths to the lightpaths under the Distinct Color Assignment (DCA) constraint and Wavelength Continuity constraint. The DCA constraint states that lightpaths on the same fiber link must be assigned with the distinct colors. The Wavelength Continuity constraint states that a lightpath must occupy the same wavelength along the links that it spans. The Wavelength Continuity constraint could be relaxed by deploying the wavelength conversion that allows the lightpaths to switch to any wavelengths at the links.

We maximize the performance in term of the traffic throughput or the successful routed traffic and we minimize the cost in term of the number of transceivers or number of lightpaths (one lightpath requires two transceivers, one at each end). Besides, we minimize the average propagation delay and the average hop count of the lightpaths. In the high-speed networks, the propagation delay is a dominance delay while the queuing delay is neglected. The lengthy fiber causes not only a considerable delay but also the impairments such as the noise accumulation, fiber chromatic dispersion, polarization, mode dispersion and fiber non linearities. Also the more hop counts of lightpath in the physical topology, the more switch ports at the intermediate nodes.

We assume that all nodes are capable of grooming low speed traffics to the available capacity of a lightpath as many as needed and a transceiver is free tuned to any wavelengths. The architecture of a node is presented in [1]. We do not allow the demultiplexing of $\mathrm{OC}-\mathrm{x}$ lower than its capacity when routing through the optical network. A bifurcate route of multiple streams through the network is allowed. Mathematical formulation of the virtual topology design related parameters, variables, constraints and objectives are given below:

\section{Given Parameters:}

- $\quad N$ : Number of Optical nodes.

- $W$ : Number of Wave lengths that can be multiplexed on a single fiber i.e., the DWDM capacity.

- $\quad T_{i}$ : Number of Transmitters at node $i ; T_{i} \geq 1 \forall i$

- $\quad R_{i}$ : Number of Receivers at node $i ; R_{i} \geq 1 \forall i$

- $K$ : Number of shortest paths or alternative routes.

- $\quad P$ : Physical topology matrix.

$$
P=\left[P_{m n} ; m, n=1,2, \ldots, N\right]_{N \times N},
$$

where $\mathrm{P}_{m n}$ is the number of fibers between node $m$ and node $n$. Note that: $\mathrm{P}_{m n}=\mathrm{P}_{n m}$.

- $\quad \Lambda: N \times N$ traffic demand matrix

$$
\Lambda^{x}=\left[\Lambda_{s d}^{x} ; s, d=1,2, \ldots, N\right]_{N \times N},
$$

where $\Lambda_{s d}^{x}$ is the demand of low speed streams, OC- $x$, between node $s$ and node $d ; x \in\{1,3,12\}$.

- $d_{m n}$ : Propagation delay weight factor on fiber link from node $m$ to $n$. The $d_{m n}$ relies on the length of fiber.

\section{Variables:}

- $\sigma_{i j, m n, w}^{k}:$ An indicator representing the existence of lightpath where

$$
\sigma_{i j, m n, w}^{k}= \begin{cases}1, & \begin{array}{l}
\text { if there exists a lightpath from } i \text { to } j \\
\text { being routed through fiber link } m n \text { on } \\
\text { the } k^{\text {th }} \text { path in } K \text { and using wavelength } \\
w \text { in } W .
\end{array} \\
0, & \text { otherwise. }\end{cases}
$$

- $V_{i j}$ : The number of lightpaths from node $i$ to node $j$ in the virtual topology.

- $\quad \lambda_{s d, i j}^{x}$ : The number of OC- $x$ streams from node $s$ to node $d$ being routed on the lightpath $V_{i j}$.

- $C$ : The capacity of a lightpath e.g., $C=48$ for OC-48.

- $S_{s d}^{x}:$ The number of OC- $x$ streams requested from node $s$ to node $d$ that are successfully routed. The traffic is blocked if $S_{s d}^{x}<\Lambda_{s d}^{x}$.

\section{Constraints:}

- Traffic (Multicommodity-flow equations for lightpath routing):

$$
\begin{array}{cl}
\sum_{m} \sum_{k} \sum_{w} \sigma_{i j, m v, w}^{k}=\sum_{n} \sum_{k} \sum_{w} \sigma_{i j, v n, w}^{k} & \text { if } v \neq i, j \quad \forall i j \\
\sum_{n} \sum_{k} \sum_{w} \sigma_{i j, i n, w}^{k}=V_{i j} & \forall i j \\
\sum_{m} \sum_{k} \sum_{w} \sigma_{i j, m j, w}^{k}=V_{i j} & \forall i j
\end{array}
$$


Equation (1) allows a lightpath to have any wavelengths in each link along the path. It implies that wavelength converters are available at all nodes. Otherwise, if we need to preserve the Wavelength Continuity rule, Equations (1), (2) and (3) will become Equations (4), (5) and (6) respectively.

$$
\begin{gathered}
\sum_{m} \sum_{k} \sigma_{i j, m v, w}^{k}=\sum_{n} \sum_{k} \sigma_{i j, v n, w}^{k} \text { if } \quad v \neq i, j \quad \forall i j, w \\
\sum_{n} \sum_{k} \sigma_{i j, i n, w}^{k}=V_{i j} \quad \forall i j, w \\
\sum_{m} \sum_{k} \sigma_{i j, m j, w}^{k}=V_{i j} \quad \forall i j, w
\end{gathered}
$$

- Wavelength Constraints:

$$
\sum_{i j} \sum_{k} \sigma_{i j, m n, w}^{k} \leq P_{m n} \quad \forall m n, w
$$

Equation (7) ensures that distinct channels (lightpaths) on the same fiber link cannot be assigned the same wavelength. Note that lightpaths $i j$ using different fibers between link $m n$ are known to be on different paths in $K$.

- Resources:

$$
\begin{gathered}
\sum_{j} V_{i j} \leq T_{i} \quad \forall i \\
\sum_{i} V_{i j} \leq R_{j} \quad \forall j
\end{gathered}
$$

Equation (8) ensures that the number of lightpaths originate from node $s$ is not greater than the number of transmitters at that node. Likewise Equation (9) ensures that the number of lightpaths terminated at node $d$ is not greater than the number of receivers at that node.

- Traffic (Multicommodity-flow equations for traffic routing):

$$
\begin{aligned}
\sum_{i} \lambda_{s d, i v}^{x}= & \sum_{j} \lambda_{s d, v j}^{x} \quad \text { if } \quad v \neq s, d \quad \forall s d, x \\
& \sum_{j} \lambda_{s d, s j}^{x}=S_{s d}^{x} \quad \forall s d, x \\
& \sum_{i} \lambda_{s d, i d}^{x}=S_{s d}^{x} \quad \forall s d, x
\end{aligned}
$$

$$
S_{s d}^{x} \leq \Lambda_{s d}^{x} \quad \forall s d, x
$$

- Capacity Constraint

$$
\sum_{x} \sum_{s d}\left(x \times \lambda_{s d, i j}^{x}\right) \leq V_{i j} \times C \quad \forall i j
$$

\section{Objectives:}

1) Maximize traffic throughput

$$
\operatorname{Max} \sum_{x, s d}\left(x \times S_{s d}^{x}\right)
$$

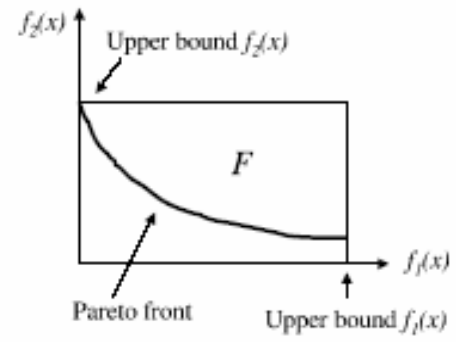

Figure 1. The pareto front and feasible solution area, $F$, of two objective functions.

2) Minimize number of transceivers or lightpaths

$$
\operatorname{Min} \sum_{i j} V_{i j}
$$

3) Minimize Average Propagation Delay (APD)

$$
\operatorname{Min} \frac{1}{\sum_{i, j} V_{i j}} \sum_{i, j} \sum_{m, n}\left(d_{m n} \sum_{k} \sum_{w} \sigma_{i j, m n, w}^{k}\right)
$$

Note that Equation (17) becomes Average Hop Count of lightpaths if $d_{m n}=1, \forall m n$.

\section{MULTI-OBJECTIVE EVOLUTIONARY ALGORITHM}

The Multi-objective Optimization (MO) or multicriteria/multiperformance/vector optimization problem involves simultaneous optimization of several competitive objectives. In MO, there is a set of optimal solutions that are 
superior (dominated) to other solutions in the search space when considering all objectives. The set of optimal solutions is known as a Pareto Optimal set. The solutions in the Pareto set are non-dominated solutions comparing to each other. Most MO algorithms use the concept of domination to search for the Pareto Optimal set. The definition of domination is defined in Definition 1 below.

Definition1: Given " $\triangleleft$ " be the operator such that $f_{k}(\mathbf{x}) \triangleleft f_{k}(\mathbf{y})$ if a solution $\mathbf{x}=\left(x_{1}, x 2, \ldots, x_{m}\right)$ is a better solution than a solution $\mathbf{y}=\left(y_{1}, y 2, \ldots, y_{m}\right)$ for the $k^{\text {th }}$ objective and $m$ parameters (decision variables). The "better" means "less than" in case of minimization or means "greater than" in case of "maximization".

If there are $n$ objectives, a solution $\mathbf{x}$ is said to dominate a solution $\mathbf{y}$ if

$$
\begin{gathered}
\forall i \in\{1,2, \ldots, n\}: f_{i}(\mathrm{x}) \triangleright f_{i}(\mathrm{y}) \wedge \\
\quad \exists i \in\{1,2, \ldots, n\}: f_{i}(\mathrm{x}) \triangleleft f_{i}(\mathrm{y})
\end{gathered}
$$

In words, a solution $\mathbf{x}$ is said to dominate a solution $\mathbf{y}$ ( or $\mathbf{x}$ is non-dominated by $\mathbf{y}$ ) if 1 and 2 are true:

1) The solution $\mathbf{x}$ is no worse than $\mathbf{y}$ for all objectives,

2) The solution $\mathbf{x}$ is strictly better than $\mathbf{y}$ in at least one objective.

The plot of non-dominated solutions in Pareto set will form the Pareto front. The Pareto front for the minimization on both objective functions, $f_{1}(\mathbf{x})$ and $f_{2}(\mathbf{x})$ is shown in Figure 1 where the $F$ area denotes the feasible solutions area. Note that the Pareto front needs not be convex.

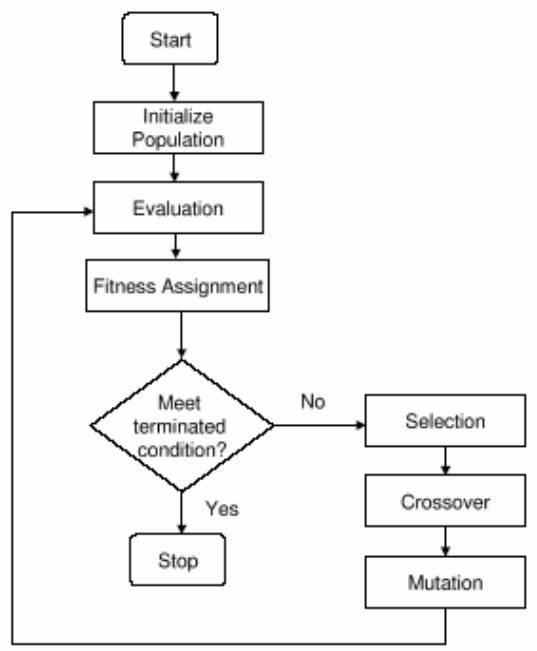

Figure 2. The MOEA-operation diagram.
There are many methods to search this set of solutions or Pareto optimal set. One of the traditional approaches is the aggregation method. It combines the objectives into a scalar function and applies the single-objective-optimization methods like a simulated annealing, a stochastic local search or a tabu search on it. The approach of combining objectives includes the weighting method [5], the constraint method [5], the goal programming [6] and the minmax method [7]. Although the aggregation methods are simple and applicable to the single objective methods but they cannot generate all members of the Pareto optimal set with non-convex Pareto front. Also the weighting factor in weighting method is quite subjective rather than straightforward and difficult to define. Moreover, each run of the algorithm will produce only a single solution. They require multiple runs to compare the domination in order to construct the Pareto optimal set.

Other methods for multi-objective optimization are the Evolutionary Algorithms called Multi-Objective Evolutionary Algorithm (MOEA) that simulates the process of natural evolution using a class of stochastic optimization methods. These methods are able to capture a Pareto optimal set in a single run. Moreover, they are less susceptible to the shape or continuity of the Pareto fronts. Therefore it is able to search on a problem with non-convex Pareto front. Most MOEAs are modified from the single-objective evolutionary algorithms like Genetic Algorithm. Therefore, the operations of MOEA include a population initialization, an evaluation, a fitness assignment, a reproduction (selection), a crossover and a mutation. Figure 2 shows the MOEA-operation diagram.

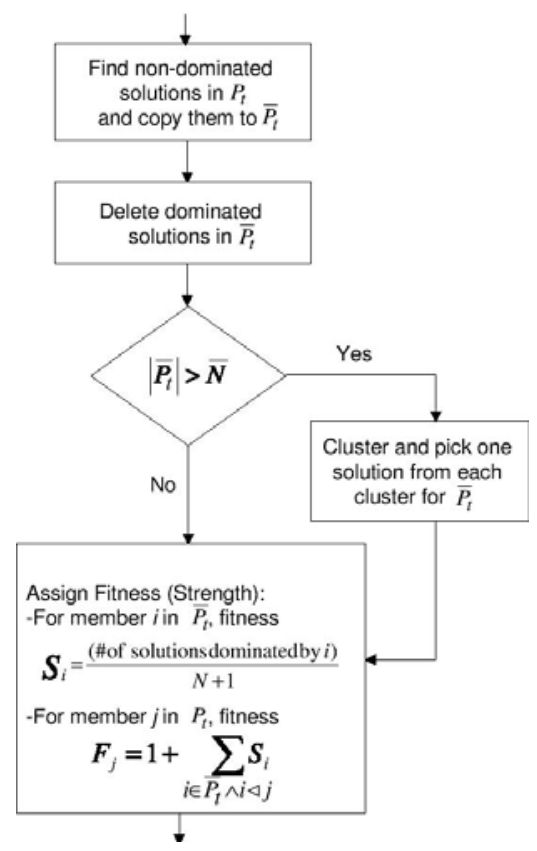

Figure 3. The SPEA fitness assignment diagram. 
There are several approaches in the MOEA such as the Vector Evaluated Genetic Algorithm (VEGA) [8], the MultiObjective Genetic Algorithm (MOGA) [9], the Nondominated Sorting Genetic Algorithm (NSGA) [10], Strength Pareto Evolutionary Algorithm (SPEA) [11] and Niched Pareto Genetic Algorithm (NPGA) [12] but the SPEA is the most efficient one.

\section{MOEA FOR THE TRAFFIC GROOMING}

We implemented MOEA for a traffic grooming problem using SPEA which outperforms other MOEAs as stated in [11]. The SPEA is a Pareto-based approach like MOGA, NSGA and NPGA which implement the Pareto-based fitness assignment strategy to determine the reproduction probability of each individual. The SPEA maintains the set of nondominated solutions in the separated population. Hence, there are two populations, the dominated population $P_{t}$ of size $N$ and the external non-dominated population $\bar{P}_{t}$ with the limited size of $\bar{N}$, where $t$ denotes the $t^{\text {th }}$ generation. The SPEA maintains the external population of the Pareto optimal solutions to reserve the elites in every generation. It assigns scalar fitness called strength (i.e., $S_{i}$ in Figure 3) to external population $\overline{P_{t}}$ and assigns fitness (i.e., $F_{j}$ in Figure 3 ) to dominated population $P_{t}$ based on the domination and strength values. The size of non-dominant solutions in Pareto front, $\bar{N}$, is controlled by a clustering algorithm such that less crowded elites are kept for the next generation. The selection or reproduction is a binary tournament selection procedure with better fitness values. A crossover operator and a mutation operator are applied to the mating pool as usual. We summarize the SPEA fitness assignment sub-operations in Figure 3. The first step is to find the non-dominated solutions within $P_{t}$ and then copy them to the external nondominated population $\overline{P_{t}}$. Thereafter, some of the copied solutions may dominate the existing solutions in $\overline{P_{t}}$. Therefore the dominated solutions found in $\bar{P}_{t}$ must be deleted. This is to ensure the non-dominated solutions are kept in $\bar{P}_{t}$ and carried through the next generation (elitist property). In the next step, it maintains the size of $\bar{P}_{t}$ i.e., the number of solutions in $\overline{P_{t}}$ must be less than or equal to $\bar{N}$. Otherwise, the clustering algorithm is performed to reduce the size of $\bar{P}_{t}$ to $\bar{N}$. The clustering algorithm is based on the Euclidean distance. At the beginning, each solution itself is a cluster. Thereafter, two clusters with the minimum clusterdistance are merged into a bigger cluster. The merging is repeated until the number of clusters is reduced to $\bar{N}$. Next the number of solutions in each cluster must be reduced to one. The algorithm keeps the solution which has the minimum average distance from other solutions in the cluster and deletes the others in that cluster. After the size of $\bar{P}_{t}$ is reduced, the fitness called Strength is assigned to each solution in $\bar{P}_{t}$ and $P_{t}$ by the equations shown in the final block of Figure 3. Note that we consider a two-objective minimization problem, so a smaller fitness represents a better solution. The detail of the SPEA algorithm can be found in [11] and [14]. We retain the main algorithm of the SPEA but modify the objective functions to exploit it for the traffic grooming problem.

For the traffic grooming problem, the solution is a virtual topology with the objectives that maximize the throughput in Equation (15), minimize the APD in Equation (17) and minimize the number of transceivers in Equation (16). These competitive objectives are evaluated (by the definition of domination) and incorporated in the fitness assignment process as shown in Figure 3. For example, if we consider Equation (15) and Equation (16), a solution $x$ is said to dominate a solution $y$ if 1 and 2 are true:

1) The solution $x$ has equal or higher throughput than that of $y$ and uses equal or lower number of transceivers than that of $y$.

2) There exists one objective that the solution $x$ is better (not just equal) than that of $y$ (in term of higher throughput or lower number of transceivers).

We create the Pareto front of the traffic grooming problem with a shape that is the same as in Figure 1. Thus we modify the problem by converting the first objective to a minimization objective by minimizing the negative value of the throughput instead. So it becomes two-objective minimization problem. The genome or individual is an encoded virtual topology which is presented here as our solution. We encode the solution by the string of $N \times(N-1)$ elements, where $N$ is the total number of nodes in the optical network. The first element presents the lightpaths from node 0 to node 1 , the second element for node 0 to node 2 , the third element for node 0 to node 3 and so forth. Each element contains $T_{i}$ unit of path indexes from node $i$ to node $j$ where $i \neq j$ and $T_{i}$ is the number of transmitters at node $i$. Each path index presents the physical route of a lightpath. If the path index $k^{\text {th }}=0$, there is no lightpath on that transmitter. Otherwise, the lightpath is using the $k^{\text {th }}$ path. The path index between node $i$ and $j$ is pre-calculated based on the $\mathrm{K}$-shortest paths over a physical topology or by the random alternative 
paths. The set of shortest paths or alternative paths is calculated in advance with regard to the given physical topology. Figure 4 shows an example of genome encoding where the number of transmitters is equal to 3 . There is one lightpath from node 0 to 1 using the first transmitter with path index $=1$

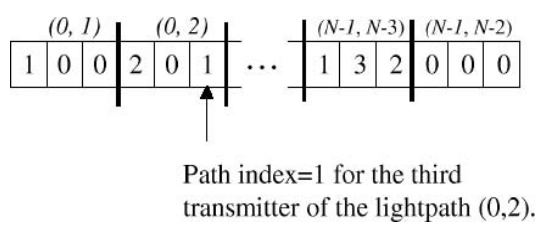

Figure 4. An example of genome encoding.

After generating the virtual topology, we adjust the number of transmitters to satisfy the resource constraints by eliminating a lightpath which occupies the lightest traffic at each source node until the constraint is satisfied. We do the same process with the number of receivers' constraints at each destination node.

Then we perform the Traffic Routing and the Wavelength Assignment separately. Since the genome does not encode the traffic route and the assigned wavelength (otherwise, the size of genome and the search space will be too large), we route the traffic and assign wavelength based on our heuristics. We route the traffic over the virtual topology of genome using the shortest path algorithm. The routing begins with the OC-12 demands first, followed by OC-3 demands and $\mathrm{OC}-1$ demands. Bifurcate routing is allowed only in each demand level itself (i.e., an OC-12 demand cannot break into four OC-3s to route separately.) We route traffic streams as many as possible over a single hop of lightpath first. The remaining traffic after that is routed over multiple hops of lightpaths.

We number the entire wavelengths (colors) and keep them in a stack one for each fiber link. If the wavelength continuity is considered, we assign the lowest available number (comparing to every stack that is on the lightpaths pan) to the lightpath that has the maximum hop-count (physical hop) first and so on. Otherwise, without wavelength continuity constraint, we assign the lowest color number found in each link's stack to the lightpath. We set a penalty function to the genome if any of the lightpaths in the genome cannot assigna color. That is to downgrade the fitness value of genome and cause it to be eliminated in the next generation. The fitness is set by the successfully routed traffic (in negative value) routed over the virtual topology and the number of transceivers (i.e., the number of lightpaths). The result of the SPEA is the external population representing the Pareto front. It contains all Pareto Optimal solutions. Another benefit of MOEA over ILP is that MOEA can generate the set of non- dominate solutions in a single run while ILP generates the solution one at a time. Moreover, when solving two objectives in ILP, we need to set one objective as a constraint while optimizing the other one. The MOEA generates the Pareto front or set of non-dominate solutions. One needs a policy to pick up a preferred solution along the Pareto front. We do not present the policy how to pick a right grooming solution on the Pareto front since we do not define the weights of the objectives. However we pick the solution according to the comparison criteria with the heuristics' results shown in the next section. For example, when comparing the throughput with the same number of transceivers, we pick the solution on the Pareto front that has the same (or lower) number of transceivers as with the heuristics' solutions and compare the throughput.

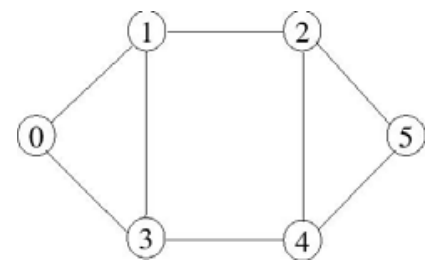

Figure 5. The 6-node network topology.

Table 1. Traffic matrix of OC-1 streams.

\begin{tabular}{|c|cccccc|}
\hline Node & 0 & 1 & 2 & 3 & 4 & 5 \\
\hline 0 & 0 & 5 & 4 & 11 & 12 & 9 \\
1 & 0 & 0 & 8 & 5 & 16 & 6 \\
2 & 14 & 12 & 0 & 9 & 6 & 16 \\
3 & 4 & 11 & 15 & 0 & 1 & 5 \\
4 & 10 & 2 & 3 & 3 & 0 & 9 \\
5 & 2 & 1 & 8 & 15 & 13 & 0 \\
\hline
\end{tabular}

Table 2. Traffiic matrix of OC-3 streams.

\begin{tabular}{|c|llllll|}
\hline Node & 0 & 1 & 2 & 3 & 4 & 5 \\
\hline 0 & 0 & 6 & 2 & 1 & 5 & 4 \\
1 & 8 & 0 & 8 & 6 & 7 & 8 \\
2 & 1 & 3 & 0 & 0 & 2 & 7 \\
3 & 5 & 7 & 3 & 0 & 2 & 6 \\
4 & 6 & 4 & 5 & 0 & 0 & 2 \\
5 & 5 & 4 & 4 & 2 & 0 & 0 \\
\hline
\end{tabular}

Table 3. Traffiic matrix of OC-12 streams.

\begin{tabular}{|c|llllll|}
\hline Node & 0 & 1 & 2 & 3 & 4 & 5 \\
\hline 0 & 0 & 1 & 1 & 1 & 0 & 0 \\
1 & 1 & 0 & 1 & 1 & 0 & 2 \\
2 & 0 & 1 & 0 & 2 & 1 & 0 \\
3 & 2 & 0 & 2 & 0 & 2 & 0 \\
4 & 1 & 2 & 0 & 2 & 0 & 1 \\
5 & 1 & 1 & 2 & 2 & 2 & 0 \\
\hline
\end{tabular}




\section{SIMULATION AND NUMERICAL RESULTS}

In this section, the performance of our approach method is presented. We set up the experiment using the 6-node network and traffic demands found in [1]. The 6-node network physical topology is shown in Figure 5 and the traffic demand matrices are shown in Tables 1-3.

We compare the result with two heuristics, the MST (Maximizing Single-Hop Traffic) and the MRU (Maximizing Resource Utilization), presented in [1]. The MST intends to construct the lightpaths between node pairs ordered by demand from the highest one. The MRU intends to construct the lightpaths between node pairs ordered by the resource utilization value which is a proportion between node pair traffic and its hop count. We modified their heuristics to include the number of transceivers and the average hop count of lightpath in the results for our comparison. The heuristics' results are varied from the results in [1] because the shortest path algorithm may select the different paths if there are more than one shortest paths with the same hop counts. We re-ran their heuristics with their traffic demand matrices, OC-1, OC3 and OC-12, to calculate the number of transceivers for our comparison. Also we found out that the ILP results in [1] is $5 \%$ less than the optimum. That makes our approach better than that of the ILP presented in [1]. The simulations are varied by the resource constraints i.e., the maximum transceivers per node, $T$, and the maximum wavelengths per fiber, $W$. We ran the MOEA with the population size $=100$, the probability of crossover $=0.8$, the probability of mutation $=0.01$, the number of generations $=5000$ and the alternative paths $\mathrm{K}=4$.

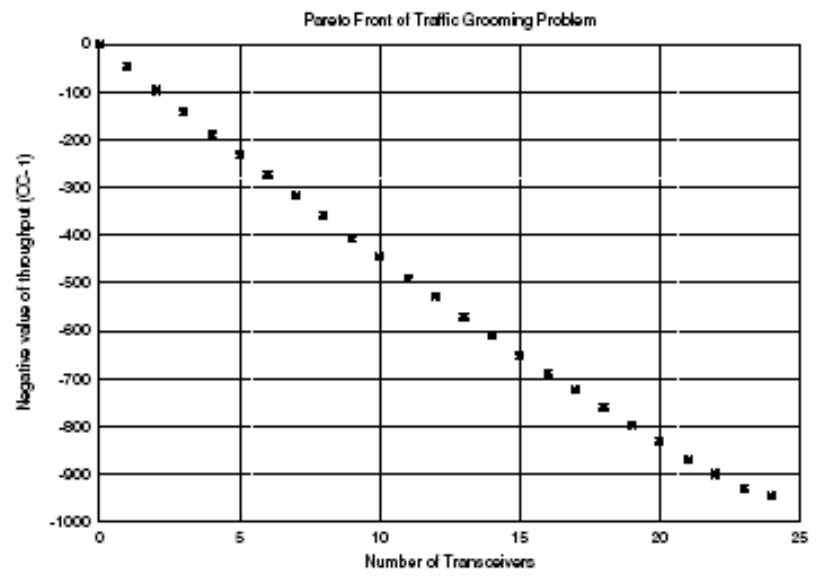

Figure 6. The Pareto front of grooming using MOEA where $T=4$ and $W=4$.
First, we show the Pareto front of the grooming problem. Since the Pareto front in Figure 1 is between two minimization objective functions, we change the throughput to its negative value. Therefore, the graph in Figure 6 is the Pareto front of grooming problem that minimizes the (negative) throughput and minimizes the number of transceivers. Figure 6 is the MOEA grooming of multi-hop traffic with wavelength continuity constraint where $\mathrm{T}=4$ and $\mathrm{W}=4$.

Next, we compare the results among the MOEA with wavelength conversion (WC), the MOEA without WC, the MST and the MRU in term of the maximum multi-hop throughput as shown in Table 4. It illustrates that the MOEA with WC outperform other approaches (except the MOEA with $\mathrm{WC}$ at $\mathrm{T}=3, \mathrm{~W}=3$, which the better solution may not yet be found within 5000 generations). Since the wavelength conversion allows the flexible routing of lightpaths, i.e., relaxes the wavelength continuity constraint, it produces better results. However, the MOEA with the wavelength continuity is still superior to the heuristic approaches.

Next, we compare the results of the MOEA, the MST and the MRU in term of multi-hop throughput with the same number of transceivers (with wavelength continuity constraint). Since the MOEA maximizes the throughput and minimizes the number of transceivers simultaneously, it produces better results (higher throughput) than that of the heuristic approaches as shown in Table 5.

Next, we perform the MOEA (with WC) with three objective functions to maximize the traffic throughput, to minimize the number of lightpaths, and to minimize the APD. We assume that $d_{m n}=1 \forall m, n$. Therefore, the APD is equivalent to the average hop counts of the lightpath. Again we compare the results with the MST and the MRU. We pick solutions in the Pareto front of MOEA results to compare with heuristics in term of the three objectives as shown in Table 6 . We can see that MOEA provides better and higher throughput, lower number of transceivers and lower APD or equivalent results comparing to the heuristics.

Finally, we show the processing time of the MOEA running on the Linux, Pentium based PC $500 \mathrm{MHz}$. In the worst case of $\mathrm{W}=3, \mathrm{~T}=7$, the MOEA with wavelength conversion takes 306 seconds and the MOEA without wavelength conversion takes 301 seconds. In the worst case of $\mathrm{W}=4$, $\mathrm{T}=5$, the MOEA with wavelength conversion takes 317 seconds and the MOEA without wavelength conversion takes 316 seconds. 
Table 4. Maximum throughput comparison.

\begin{tabular}{|c|c|c|c|c|c|c|c|c|}
\hline & \multicolumn{2}{|c|}{ MOEA (with WC) } & \multicolumn{2}{|c|}{ MOEA } & \multicolumn{2}{|c|}{ MST } & \multicolumn{2}{|c|}{ MRU } \\
\hline & Throughput & \#Transceivers & Throughput & \#Transceivers & Throughput & \#Transceivers & Throughput & \#Transceivers \\
\hline $\mathrm{T}=3 \mathrm{~W}=3$ & OC-739 & 18 & OC-751 & 18 & OC-69917 & 17 & OC-65017 & 17 \\
\hline $\mathrm{T}=4 \mathrm{~W}=3$ & OC-937 & 24 & OC-934 & 24 & OC-89622 & 22 & OC-90923 & 23 \\
\hline $\mathrm{T}=5 \mathrm{~W}=3$ & OC-969 & 26 & OC-969 & 26 & OC-93524 & 24 & OC-92024 & 24 \\
\hline $\mathrm{T}=7 \mathrm{~W}=3$ & OC-969 & 26 & OC-969 & 26 & OC-93524 & 24 & OC-92024 & 24 \\
\hline $\mathrm{T}=3 \mathrm{~W}=4$ & OC-749 & 18 & OC-739 & 18 & OC-69917 & 17 & OC-65017 & 17 \\
\hline $\mathrm{T}=4 \mathrm{~W}=4$ & OC-946 & 24 & OC-946 & 24 & OC-92223 & 23 & OC-92023 & 23 \\
\hline $\mathrm{T}=5 \mathrm{~W}=4$ & OC-988 & 25 & OC-988 & 26 & OC-98828 & 28 & OC-98828 & 28 \\
\hline
\end{tabular}

Table 5. Throughput and number of transceivers comparison.

\begin{tabular}{|c|c|c|c|c|c|c|}
\hline & \multicolumn{2}{|c|}{ MOEA } & \multicolumn{2}{|c|}{ MST } & \multicolumn{2}{|c|}{ MRU } \\
\hline & Throughput & \#Transceivers & Throughput & \#Transceivers & Throughput & \#Transceivers \\
\hline $\mathrm{T}=3 \mathrm{~W}=3$ & OC-712 & 17 & OC-699 & 17 & OC-650 & 17 \\
\hline $\mathrm{T}=4 \mathrm{~W}=3$ & $\begin{array}{l}\text { OC-896 } \\
\text { OC-920 }\end{array}$ & $\begin{array}{l}22 \\
23\end{array}$ & OC-896 & 22 & OC-909 & 23 \\
\hline $\mathrm{T}=5 \mathrm{~W}=3$ & OC-944 & 24 & OC-935 & 24 & OC-920 & 24 \\
\hline $\mathrm{T}=7 \mathrm{~W}=3$ & OC-956 & 24 & OC-935 & 24 & OC-920 & 24 \\
\hline $\mathrm{T}=3 \mathrm{~W}=4$ & $\mathrm{OC}-714$ & 17 & OC-699 & 17 & $\mathrm{OC}-650$ & 17 \\
\hline $\mathrm{T}=4 \mathrm{~W}=4$ & OC-931 & 23 & OC-922 & 23 & OC-920 & 23 \\
\hline $\mathrm{T}=5 \mathrm{~W}=4$ & OC-988 & 26 & OC-988 & 28 & OC-988 & 28 \\
\hline
\end{tabular}

Table 6. Throughput, number of transceivers, and APD comparison.

\begin{tabular}{|c|c|c|c|c|c|c|c|c|c|}
\hline & \multicolumn{3}{|c|}{ MOEA } & \multicolumn{3}{|c|}{ MST } & \multicolumn{3}{|c|}{ MRU } \\
\hline & Throughput & \#Transceivers & APD & Throughput & \#Transceivers & APD & Throughput & \#Transceivers & APD \\
\hline \multirow{2}{*}{$\mathrm{T}=3 \mathrm{~W}=3$} & 707 & 17 & 1.29 & \multirow{2}{*}{699} & \multirow{2}{*}{17} & \multirow{2}{*}{1.41} & \multirow{2}{*}{650} & \multirow{2}{*}{17} & \multirow{2}{*}{1.12} \\
\hline & 659 & 17 & 1.12 & & & & & & \\
\hline \multirow[t]{2}{*}{$\mathrm{T}=4 \mathrm{~W}=3$} & 896 & 22 & 1.45 & \multirow[t]{2}{*}{896} & \multirow[t]{2}{*}{22} & \multirow[t]{2}{*}{1.45} & \multirow[t]{2}{*}{909} & \multirow[t]{2}{*}{23} & \multirow[t]{2}{*}{1.30} \\
\hline & 909 & 23 & 1.30 & & & & & & \\
\hline $\mathrm{T}=5 \mathrm{~W}=3$ & 947 & 24 & 1.33 & 935 & 24 & 1.42 & 920 & 24 & 1.33 \\
\hline $\mathrm{T}=7 \mathrm{~W}=3$ & 947 & 24 & 1.33 & 935 & 24 & 1.42 & 920 & 24 & 1.33 \\
\hline \multirow[t]{2}{*}{$\mathrm{T}=3 \mathrm{~W}=4$} & 712 & 17 & 1.41 & \multirow[t]{2}{*}{699} & \multirow[t]{2}{*}{17} & \multirow[t]{2}{*}{1.53} & \multirow[t]{2}{*}{650} & \multirow[t]{2}{*}{17} & \multirow[t]{2}{*}{1.12} \\
\hline & 650 & 17 & 1.12 & & & & & & \\
\hline $\mathrm{T}=4 \mathrm{~W}=4$ & 926 & 23 & 1.43 & 922 & 23 & 1.48 & 920 & 23 & 1.39 \\
\hline $\mathrm{T}=5 \mathrm{~W}=4$ & 988 & 26 & 1.46 & 988 & 28 & 1.46 & 988 & 28 & 1.46 \\
\hline
\end{tabular}

\section{Conclusion and Future Work}

In this paper, we proposed the Multi-Objective Evolutionary Algorithm approach for the traffic grooming problem in WDM Optical mesh networks. It maximizes the throughput, minimizes the number of transceivers, and minimizes the average propagation delay at the same time. We formulated the traffic grooming problem and proposed the MOEA method to the traffic grooming problem with the encoding, routing, and wavelength assignment schemes. We plotted the two objectives graph to show the Pareto front of the grooming problem and then we ran the simulation to compare the results with the results of heuristic approaches, MST, and MRU. The comparison results showed that MOEA performs better in any cases than that of two heuristics with the acceptable processing time. The MOEA can be applied to the network revenue model to reduce other costs while increasing the performance since the number of objective functions can be extended. 


\section{References}

[1] K. Zhu and B. Mukherjee, "Traffic Grooming in an Optical WDM Mesh Network, "IEEE JSAC, Vol. 20, No. 1, January 2002, pp. 122-133.

[2] V. R. Konda and T. Y. Chow, "Algorithm for Traffic Grooming in Optical Networks to Minimize the Number of Transceivers," IEEE 2001 High Performance Switching and Routing Workshop, pp. 218-221.

[3] S. Thiagarajan and A. K. Somani, "Capacity Fairness of WDM Networks with Grooming Capabilities," Optical Networks Magazine, Vol. 2, No. 3, May/June 2001, pp. 24-31.

[4] S. Thiagarajan and A. K. Somani, "A Capacity Correlation Model for WDM Networks with Constrained Grooming Capabilities," IEEE International Conference on Communications 2001, Vol 5, pp.1592-1596.

[5] J. L. Cohon, Multi objective Programming and Planning, New York, Academic Press, 1978.

[6] R. E. Steuer, Multiple Criteria Optimization: Theory, Computation and Application, New York, Wiley, 1986.

[7] J. Koskietal, "Multicriterion Optimization in Structural Design, New Directions in Optimum Structural Design," Wiley, 1984, pp. 483-503.

[8] J. D. Schaffer, "Multiple Objective Optimization with Vector Evaluated Genetic Algorithms, In Genetic Algorithms and their Application," Proceedings of the First International Conference on Genetic Algorithms, Lawrence Erlbaum, 1985, pp. 93-100.

[9] C. M. Fonseca and P. J. Fleming, "Genetic Algorithms for Multiobjective Optimization: Formulation, Discussion and Generalization," Proceedings of the Fifth International Conference on Genetic Algorithms, 1993, pp. 416-423.

[10] N. Srinavas and K. Deb, "Multiobjective Optimization using Nondominated Sorting in Genetic Algorithms," Evolutionary Computation, Vol 2(3), 1994, pp. 221248.

[11] E. Zitzlerand L. Thiele, "Multiobjective Evolutionary Algorithms: A Comparative Case Study and the Strength Pareto Approach," IEEE Transactions on Evolutionary Computation, 3(4), November1999, pp. 257-271.
[12] J. Hornetal, "A Niched Pareto Genetic Algorithm for Multiobjective Optimization," Proceedings of the First IEEE Conference on Evolutionary Computation, Vol 1, June 1994, pp. 82-87.

[13] K. Zhu and B. Mukherjee, "Traffic Grooming in an Optical WDM Mesh Network," Selected Areas in Communications, IEEE Journal on, Vol 20 Issue 1, Jan 2002, pp. 122-133.

[14] K. Deb, Multi-Objective Optimization using Evolutionary Algorithms, John Wiley \& Sons, Inc., New York, 2001.

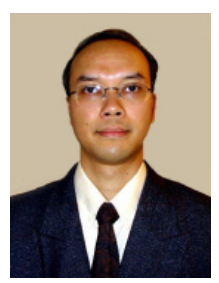

Passakon Prathombutr was born on August 8, 1968 in Nakhon Sawan, Thailand. He attended Chiang Mai University and received a B.S. degree in Physics (HONS) in 1990. He pursued the higher degree and graduated a M.S. degree in Computer Science from Chulalongkorn University, Thailand in 1993. While he became the researcher at the National Electronic and Computer Technology Center (NECTEC) Ministry of Science, Thailand, he received the government scholarship to study the Ph.D. program in data communication field in the United States. He attended Oklahoma State University and graduated in 1998 with another M.S. degree in Computer Science. He continued his studies by pursing an interdisciplinary Ph.D. in Software Architecture and Computer Networking at University of Missouri Kansas City and graduated the Ph.D. program in December 2003. Now he acts as a director of the Next Generation Internet project at NECTEC, Thailand. His research areas include the IPv6, Network Security and Optical Networking. 